Quim. Nova, Vol. 34, No. 6, 1074-1078, 2011

\title{
CONSTANTES DE ACIDEZ DE ÁCIDOS DIPRÓTICOS A PARTIR DE TITULAÇÕES POTENCIOMÉTRICAS: ILUSTRAÇÃO DOS PRINCÍPIOS DO CÁLCULO ATRAVÉS DA CONSTRUÇÃO DE UM ALGORITMO MUITO SIMPLES
}

\section{Elsa M. Gonçalves}

Departamento de Química e Bioquímica, Faculdade de Ciências, Universidade de Lisboa, 1649-016 Lisboa / Instituto Politécnico de Setúbal, ESTBarreiro, Rua Américo da Silva Marinho, 2839-001 Lavradio, Portugal

António C. L. Conceição*

Centro de Química Estrutural, Complexo Interdisciplinar, Instituto Superior Técnico, 1049-001 Lisboa, Portugal

Recebido em 3/8/10; aceito em 14/12/10; publicado na web em 29/3/11

\begin{abstract}
ACIDITY CONSTANTS OF DIPROTIC ACIDS FROM POTENTIOMETRIC TITRATIONS: PRINCIPLES OF THE CALCULATION ILLUSTRATED THROUGH THE CONSTRUCTION OF A SIMPLE ALGORITHM. This work describes the creation of an very simple calculation algorithm, based in basic chemical and mathematic principles, for the calculation of weak diprotic acid dissociation constants as, for example, amino acids, from potentiometric titrations. For an easier understanding of the algorithm the logical reasoning of this calculus is schematized in a diagram of blocks. In the second part of the work the algorithm is applied to an Excel calculation sheet to determine the dissociation constants of Nicotinic Acid and Glycine, from the respective potentiometric titration curves. The values obtained using this algorithm are compared with those estimated by Hyperquad2008 (program generally used for this type of calculus) and also with the values of a stability constants database.
\end{abstract}

Keywords: dissociation constants; potentiometric titrations; algorithm.

\section{INTRODUÇÃO}

Este trabalho destina-se aos alunos de Química dos primeiros anos do ensino superior, encontra-se detalhadamente explicado e apenas envolve conceitos básicos. O intuito deste trabalho é ajudar os alunos a perceber, principalmente do ponto de vista químico, o funcionamento dos programas comerciais usados para o cálculo de constantes de equilíbrio determinadas por potenciometria.

As constantes de dissociação dos ácidos fracos são uma medida da sua força em solução e definem-se como constantes de equilíbrio para a reação de dissociação dos ácidos em solução. ${ }^{1} \mathrm{O}$ pH resultante da dissociação de um ácido fraco depende do valor dessa constante e o seu conhecimento permite a preparação de soluções tampão de $\mathrm{pH}$. Para saber mais sobre a importância da determinação das constantes de equilíbrio, consultar, por exemplo, o livro do Martell e Motekaitis. ${ }^{2}$

Um programa, de utilização muito generalizada, para calcular as constantes de equilíbrio a partir de dados de titulações potenciométricas é o Hyperquad2008. ${ }^{3,4}$ Existem muitos mais programas para o cálculo das constantes de equilíbrio a partir de dados de titulações potenciométricas. ${ }^{4}$

\section{Conceitos básicos}

Para calcular as constantes de equilíbrio de um ácido diprótico começamos por considerar os equilíbrios que se estabelecem quando dissolvemos o ácido em água:

$$
\begin{aligned}
& \mathrm{H}_{2} \mathrm{~A}+\mathrm{H}_{2} \mathrm{O} \Leftrightarrow \mathrm{HA}^{-}+\mathrm{H}_{3} \mathrm{O}^{+} \\
& \mathrm{HA}^{-}+\mathrm{H}_{2} \mathrm{O} \Leftrightarrow \mathrm{A}^{2-}+\mathrm{H}_{3} \mathrm{O}^{+}
\end{aligned}
$$

*e-mail: antonio.conceicao@ist.utl.pt
Temos de considerar também o equilíbrio de autoprotólise da água:

$$
2 \mathrm{H}_{2} \mathrm{O} \Leftrightarrow \mathrm{H}_{3} \mathrm{O}^{+}+\mathrm{OH}^{-}
$$

Tendo em conta estes equilíbrios temos em solução as espécies seguintes:

$$
\mathrm{H}_{2} \mathrm{~A} ; \mathrm{HA}^{-} ; \mathrm{A}^{2-} ; \mathrm{OH}^{-} ; \mathrm{H}_{3} \mathrm{O}^{+}
$$

Estas espécies encontram-se relacionadas entre si pelas equações de definição das constantes de equilíbrio (Equações 1-3) e pelos balanços de massa em relação ao ácido (Equação 4) e ao hidrogénio (Equação 5):

$$
\begin{gathered}
K_{\mathrm{a} 1}=\frac{\left[\mathrm{HA}^{-}\right]\left[\mathrm{H}_{3} \mathrm{O}^{+}\right]}{\left[\mathrm{H}_{2} \mathrm{~A}\right]} \\
K_{\mathrm{a} 2}=\frac{\left[\mathrm{A}^{2-}\right]\left[\mathrm{H}_{3} \mathrm{O}^{+}\right]}{\left[\mathrm{HA}^{-}\right]} \\
K_{\mathrm{w}}=\left[\mathrm{H}_{3} \mathrm{O}^{+}\right]\left[\mathrm{OH}^{-}\right] \\
\mathrm{C}_{\mathrm{A}}=\left[\mathrm{H}_{2} \mathrm{~A}\right]+\left[\mathrm{HA}^{-}\right]+\left[\mathrm{A}^{2-}\right] \\
\mathrm{C}_{\mathrm{H}}=2\left[\mathrm{H}_{2} \mathrm{~A}\right]+\left[\mathrm{HA}^{-}\right]+\left[\mathrm{H}_{3} \mathrm{O}^{+}\right]-\left[\mathrm{OH}^{-}\right]
\end{gathered}
$$

As constantes de equilíbrio assim definidas são constantes estequiométricas válidas para uma dada força iónica. Conhecendo as concentrações totais ou analíticas do ácido, $\mathrm{C}_{\mathrm{A}}$, e do hidrogénio, $\mathrm{C}_{\mathrm{H}}$, as constantes de dissociação do ácido fraco, $K_{\mathrm{a} 1}$ e $K_{\mathrm{a} 2}$, bem como a constante de autoprotólise da água, $K_{\mathrm{w}}$, podemos calcular, através de 
um sistema de cinco equações a cinco incógnitas, a concentração de todas as espécies em solução. Quando desconhecemos as constantes de dissociação do ácido fraco ficamos com mais duas incógnitas e o sistema de equações não tem solução analítica (mais incógnitas que equações). Para a resolução deste problema começamos por determinar experimentalmente a concentração do ião hidrogénio.

\section{Determinação experimental da concentração do ião hidrogênio}

Segundo a IUPAC o pH corresponde a - $\log \left(\mathrm{H}_{3} \mathrm{O}^{+}\right)$onde $\left(\mathrm{H}_{3} \mathrm{O}^{+}\right)$ é a atividade do ião $\mathrm{H}_{3} \mathrm{O}^{+}$. Neste trabalho não medimos o $\mathrm{pH}$ mas sim a força electromotriz da célula, $E$, utilizando um eléctrodo que responde à atividade do ião hidrogénio. Para saber a concentração do ião hidrogénio temos de fazer uma titulação de calibração: titulação de um ácido forte com uma base forte. Definimos $\mathrm{p}[\mathrm{H}]$ como -log $\left[\mathrm{H}_{3} \mathrm{O}^{+}\right]$que, para a titulação de um ácido forte com uma base forte, é conhecido para qualquer ponto da curva de titulação. Com efeito, para um ácido forte $\mathrm{C}_{\mathrm{H}}=\left[\mathrm{H}_{3} \mathrm{O}^{+}\right]$, em que $\mathrm{C}_{\mathrm{H}}$ é a concentração total do hidrogénio calculada a partir da concentração inicial no vaso de titulação, da concentração da base na bureta, do volume inicial no vaso de titulação e do volume de base adicionado. Seguidamente através de uma regressão linear de $E$ vs $\mathrm{p}[\mathrm{H}]$ calculamos os parâmetros da calibração do eléctrodo; ordenada na origem, $K$, e o declive, $S$. A concentração do ião hidrogénio pode agora ser conhecida quando estamos em presença de um ácido fraco, fazendo uma titulação do ácido fraco com uma base forte, curva $E v s \mathrm{~V}_{\mathrm{B}}$, e usando os parâmetros da calibração. A concentração do ião hidrogénio é então dada por:

$$
\left[\mathrm{H}_{3} \mathrm{O}^{+}\right]=10^{\frac{K-E}{S}}
$$

Assim eliminamos do sistema uma incógnita (a concentração da espécie $\mathrm{H}_{3} \mathrm{O}^{+}$).

\section{Utilização de um algoritmo de cálculo}

Nesta fase temos ainda mais uma incógnita que o número de equações e, portanto, o sistema ainda não tem solução analítica. No caso de se tratar de um ácido monoprótico o sistema já teria solução analítica por uma equação do terceiro grau. ${ }^{5}$ Para resolver este sistema iremos utilizar o algoritmo esquematizado na Figura 1, que começa

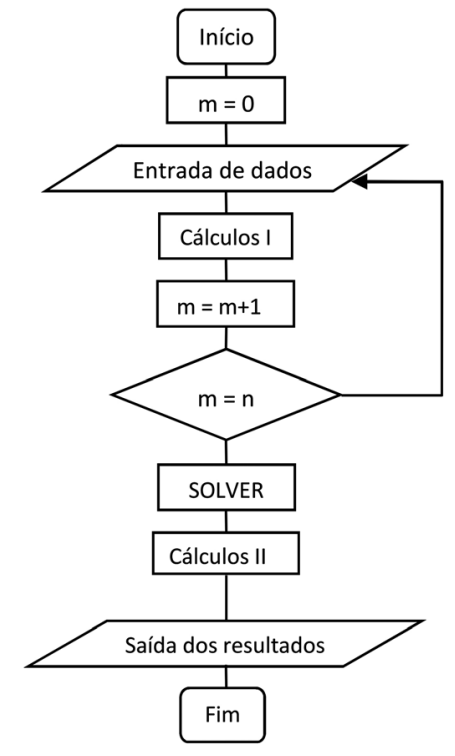

Figura 1. Diagrama lógico para o cálculo das constantes de dissociação e para a simulação da curva de titulação por arbitrar os valores das constantes de dissociação do ácido fraco e calcular a concentração total do hidrogénio dada pela Equação 5, cujo valor depende dessas constantes, e a concentração total do hidrogénio dada pela Equação 7.

$$
\mathrm{C}_{\mathrm{H}}^{*}=\frac{\left(\mathrm{C}_{\mathrm{H} 0} \times \mathrm{V}_{0}\right)-\left(\mathrm{C}_{\mathrm{B}} \times \mathrm{V}_{\mathrm{B}}\right)}{\mathrm{V}_{0}+\mathrm{V}_{\mathrm{B}}}
$$

onde $\mathrm{C}_{\mathrm{H}}^{*}$ é a concentração total do hidrogénio calculada a partir da quantidade inicial no vaso de titulação $\left(\mathrm{C}_{\mathrm{H} 0}\right)$, da concentração da base na bureta $\left(\mathrm{C}_{\mathrm{B}}\right)$, do volume inicial no vaso de titulação $\left(\mathrm{V}_{0}\right)$ e do volume de base adicionado $\left(\mathrm{V}_{\mathrm{B}}\right)$. A concentração total do hidrogénio dada por estas duas equações tem de ser igual, e será quando os valores das constantes arbitradas forem os verdadeiros.

Para efectuar estes cálculos iremos construir uma folha de cálculo no Microsoft Excel, Figura 1S, material suplementar, com base no algoritmo esquematizado na Figura 1 e que descrevemos a seguir. Este algoritmo aplica-se a sistemas que utilizam dois reagentes A e $\mathrm{H}$ e três constantes de equilíbrio, duas referentes ao ácido diprótico, mais a constante de autoprotólise da água.

\section{Construção do algoritmo de cálculo}

O algoritmo utiliza todos os pontos de uma curva de titulação e começa por dar uma solução para cada ponto, $i$, da curva de titulação, sendo o resultado final a média das $n$ soluções encontradas para os n pontos da curva de titulação utilizados. Começa-se por construir uma função SOM1 $=\sum_{\mathrm{i}=1}^{\mathrm{n}}\left(\mathrm{C}_{\mathrm{H}, i}^{*}-\mathrm{C}_{\mathrm{H}, i}\right)^{2}$, onde $\mathrm{C}_{\mathrm{H}, i}$ representa a concentração total do hidrogénio calculada a partir do balanço de massa e que depende das constantes de equilíbrio a determinar $\left(K_{\mathrm{a} 1, i}\right.$ e $\left.K_{\mathrm{a} 2, i}\right)$, dos parâmetros da calibração do eléctrodo $(K$ e $S)$, da constante de autoprotólise da água $\left(K_{\mathrm{w}}\right)$, da quantidade inicial do ácido fraco no vaso de titulação $\left(\mathrm{C}_{\mathrm{A} 0}\right)$, do potencial medido $\left(E_{i}\right)$, do volume inicial e do volume de base adicionado. Assim podemos escrever:

$\mathrm{SOM} 1=\sum_{i=1}^{\mathrm{n}}\left(\mathrm{C}_{\mathrm{H}, i}^{*}-\mathrm{C}_{\mathrm{H}, i}\right)^{2}=\mathrm{f}\left(K_{\mathrm{a} 1, i}, K_{\mathrm{a} 2, i}, K_{\mathrm{w}}, \mathrm{C}_{\mathrm{A} 0}, K, E_{i}, S, \mathrm{C}_{\mathrm{H} 0}, \mathrm{~V}_{0}, \mathrm{C}_{\mathrm{B}}, \mathrm{V}_{\mathrm{B}, i}\right)$

Para se poder calcular a função SOM1 é necessário que se atribuam valores iniciais aos parâmetros a determinar. Estes valores iniciais podem ser obtidos com a ajuda da curva calculada $\mathrm{p}[\mathrm{H}]$ em função do volume de base adicionado. Os valores iniciais das constantes terão de ser otimizados de modo a que o valor da função, SOM1, seja mínimo. Para fazer essa otimização utilizamos o Microsoft Excel Solver que usa um código de otimização não linear conhecido por Generalized Reduced Gradient (GRG2).

O diagrama lógico da Figura 1 é constituído por blocos. No bloco Entrada de dados faz-se a entrada dos dados conhecidos e dos valores arbitrados necessários ao cálculo, que são: $\mathrm{C}_{\mathrm{A} 0} ; \mathrm{V}_{0}$; volume de base adicionado no ponto $i$ da curva de titulação, $\mathrm{V}_{\mathrm{B}, i} ; \mathrm{C}_{\mathrm{B}} ; K_{w} ; S$ e $K ; \mathrm{C}_{\mathrm{H} 0}$; número de pontos da curva de titulação, n; volumes de base adicionados, $\mathrm{V}_{\mathrm{B}, 1} ; \mathrm{V}_{\mathrm{B}, 2} ; \ldots ; \mathrm{V}_{\mathrm{B}, \mathrm{n}} ;$ potenciais medidos, $E_{1} ; E_{2} ; \ldots ; E_{n}$; segue-se a entrada dos valores arbitrados, $K_{\mathrm{a} 1,1} ; K_{\mathrm{a} 1,2} ; \ldots ; K_{\mathrm{a} 1, \mathrm{n}} ; K_{\mathrm{a} 2,1} ; \ldots$; $K_{\mathrm{a} 2, \mathrm{n}}$ que são as constantes de dissociação que pretendemos calcular.

Depois da entrada dos dados temos os cálculos no bloco Cálculos I:

1 - Cálculo da concentração total do ácido fraco para cada ponto $i$ da curva de titulação:

$$
\mathrm{C}_{\mathrm{A}, i}=\frac{\mathrm{C}_{\mathrm{A} 0} \times \mathrm{V}_{0}}{\mathrm{~V}_{0}+\mathrm{V}_{\mathrm{B}, i}}
$$

2 - Cálculo da concentração do ião hidrogénio: Esta concentração 
é dada através dos potenciais medidos durante a titulação do ácido fraco com a base forte e de uma calibração (titulação ácido forte/ base forte nas mesmas condições de força iónica e de temperatura da experiência, o que permite determinar as duas constantes que relacionam o potencial do eléctrodo com a concentração do ião hidrogénio, $S$ e $K$, respectivamente, declive do eléctrodo e ordenada na origem), assim:

$$
\left[\mathrm{H}_{3} \mathrm{O}^{+}\right]_{i}=10^{\frac{K-E_{i}}{S}}
$$

3 - Cálculo da concentração da espécie $\left[\mathrm{A}^{2-}\right]_{i}$. Obtém-se substituindo as Equações 1 e 2 na Equação 4:

$$
\left[\mathrm{A}^{2-}\right]_{i}=\frac{\mathrm{C}_{\mathrm{A}, i}}{1+\frac{\left[\mathrm{H}_{3} \mathrm{O}^{+}\right]_{i}}{K_{\mathrm{a} 2, i}}+\frac{\left[\mathrm{H}_{3} \mathrm{O}^{+}\right]_{i}^{2}}{K_{\mathrm{a} 1, i} \times K_{\mathrm{a} 2, i}}}
$$

4 - Cálculo da concentração das restantes espécies. Obtêm-se por rearranjo das Equações 2 e 1:

$$
\begin{aligned}
& {\left[\mathrm{HA}_{i}\right]_{i}=\frac{\left[\mathrm{A}^{2}\right]_{i}\left[\mathrm{H}_{3} \mathrm{O}^{+}\right]_{i}}{K_{\mathrm{a} 2, i}}} \\
& {\left[\mathrm{H}_{2} \mathrm{~A}\right]_{i}=\frac{\left[\mathrm{HA}^{-}\right]_{i}\left[\mathrm{H}_{3} \mathrm{O}^{\dagger}\right]_{i}}{K_{\mathrm{a} 1, i}}}
\end{aligned}
$$

5 - Cálculo da concentração total do hidrogénio: Substituindo a Equação 3 na Equação 5, tem-se:

$$
\mathrm{C}_{\mathrm{H}, i}=2\left[\mathrm{H}_{2} \mathrm{~A}\right]_{i}+\left[\mathrm{HA}^{-}\right]_{i}+\left[\mathrm{H}_{3} \mathrm{O}^{+}\right]_{i}-\frac{K_{\mathrm{w}}}{\left[\mathrm{H}_{3} \mathrm{O}^{+}\right]_{i}}
$$

Se os valores arbitrados forem os verdadeiros a concentração calculada por esta equação tem de ser igual à concentração calculada pela equação:

$$
\mathrm{C}_{\mathrm{H}, i}^{*}=\frac{\left(\mathrm{C}_{\mathrm{H} 0} \times \mathrm{V}_{0}\right)-\left(\mathrm{C}_{\mathrm{B}} \times \mathrm{V}_{\mathrm{B}, i}\right)}{\mathrm{V}_{0}+\mathrm{V}_{\mathrm{B}, i}}
$$

Então calculamos o desvio ao quadrado entre as concentrações totais do hidrogénio dadas por estas duas equações:

$$
\mathrm{D}_{1, i}=\left(\mathrm{C}_{\mathrm{H}, i}-\mathrm{C}_{\mathrm{H}, i}^{*}\right)^{2}
$$

Repetem-se os cálculos para os n pontos da curva de titulação, e somam-se os desvios ao quadrado:

$$
\mathrm{SOM} 1=\sum_{i=1}^{\mathrm{n}}\left(\mathrm{D}_{1, i}\right)
$$

No bloco Solver utiliza-se o Microsoft Excel Solver (Figura 2Sa, material suplementar) para otimizar o valor das constantes arbitradas de modo que o somatório dos desvios ao quadrado dado pela Equação 16 seja mínimo. Todo este processo é repetido para cada ponto $i$ da curva de titulação até $i=\mathrm{n}$.

No bloco Cálculos II faz-se o cálculo dos valores médios das constantes. Uma vez obtidos os melhores valores para as constantes em cada ponto $i$ da curva de titulação calculam-se valores médios utilizando as equações:

$$
\bar{K}_{\mathrm{a} 1}=\frac{\sum_{i=1}^{\mathrm{n}} K_{\mathrm{a} 1, i}}{\mathrm{n}}
$$

$$
\bar{K}_{\mathrm{a} 2}=\frac{\sum_{i=1}^{\mathrm{n}} K_{\mathrm{a} 2, i}}{\mathrm{n}}
$$

Estes valores médios são os melhores valores para as constantes de dissociação.

O algoritmo termina com a saída dos resultados, $\bar{K}_{\mathrm{a} 1}$ e $\bar{K}_{\mathrm{a} 2}$, no bloco Saída dos resultados.

Para a zona básica da curva de titulação as Equações 13, 14 e 15 são substituídas pelas equações:

$$
\begin{gathered}
\mathrm{C}_{\mathrm{OH}, i}=\frac{K_{\mathrm{w}}}{\left[\mathrm{H}_{3} \mathrm{O}^{+}\right]_{i}}+\left[\mathrm{HA}^{-}\right]_{i}+2\left[\mathrm{~A}^{-}\right]_{i}-\left[\mathrm{H}_{3} \mathrm{O}^{+}\right]_{i} \\
\mathrm{C}_{\mathrm{OH}, i}^{*}=\frac{\left(\mathrm{C}_{\mathrm{B}} \times \mathrm{V}_{\mathrm{B}, i}\right)-\left(\mathrm{C}_{\mathrm{H} 0} \times \mathrm{V}_{0}\right)}{\mathrm{V}_{0}+\mathrm{V}_{\mathrm{B}, i}} \\
\mathrm{D}_{1, i}=\left(\mathrm{C}_{\mathrm{OH}, i}-\mathrm{C}_{\mathrm{OH}, i}^{*}\right)^{2}
\end{gathered}
$$

Todos os restantes cálculos são feitos da mesma forma que os descritos para a zona ácida. Os cálculos realizados até este ponto correspondem à primeira parte da folha de cálculo, nas colunas $\mathrm{A}$ a Q da Figura 1S, material suplementar.

Na segunda parte da folha de cálculo, nas colunas R a AA da Figura 1S do material suplementar, simulamos a curva de titulação utilizando as constantes determinadas na primeira parte. Para isso vamos utilizar um algoritmo de cálculo estruturalmente semelhante ao utilizado anteriormente, Figura 1, só diferindo no conteúdo de cada bloco que se descreve seguidamente:

No bloco Entrada de dados faz-se a entrada das constantes calculadas na primeira parte da folha de cálculo: $K_{\mathrm{a} 1,1} ; K_{\mathrm{a} 1,2} ; \ldots \ldots ; K_{\mathrm{a} 1, \mathrm{n}}$; $K_{\mathrm{a} 2,1} ; K_{\mathrm{a} 2,2} ; \ldots \ldots ; K_{\mathrm{a} 2, \mathrm{n}}$ e dos valores arbitrados: $\left[\mathrm{H}_{3} \mathrm{O}^{+}\right]_{1} ;\left[\mathrm{H}_{3} \mathrm{O}^{+}\right]_{2} ; \ldots \ldots$; $\left[\mathrm{H}_{3} \mathrm{O}^{+}\right]_{\mathrm{n}}$. Todas as outras entradas de dados necessários já foram referidas na descrição anterior.

No bloco Cálculos I começamos por calcular a concentração das espécies $\left[\mathrm{A}^{2-}\right]_{i},[\mathrm{HA}]_{i}$ e $\left[\mathrm{H}_{2} \mathrm{~A}^{+}\right]_{i}$ utilizando as Equações 10,11 e 12, respectivamente. Calculamos também a concentração total do hidrogénio, $\mathrm{C}_{\mathrm{H}, i}$, utilizando a Equação 13 para a zona ácida e a Equação 19 para a zona básica. Se os valores arbitrados forem os verdadeiros a concentração calculada pelas Equações 13 e 19 tem de ser igual à concentração calculada pelas Equações 14 na zona ácida e 20 na zona básica, respectivamente. De seguida calculamos a soma dos desvios ao quadrado entre as concentrações totais do hidrogénio (Equações 13 e 14) e as concentrações totais do ião hidróxido (Equações 19 e 20) dada pela equação:

$$
\mathrm{D}_{2, i}=\left(\mathrm{C}_{\mathrm{H}, i}-\mathrm{C}_{\mathrm{H}, i}^{*}\right)^{2}+\left(\mathrm{C}_{\mathrm{OH}, i}-\mathrm{C}_{\mathrm{OH}, i}^{*}\right)^{2}
$$

Repetem-se os cálculos para todos os n pontos da curva de titulação, e somam-se os desvios ao quadrado:

$$
\mathrm{SOM} 2=\sum_{i=1}^{\mathrm{n}}\left(\mathrm{D}_{2, i}\right)
$$

No bloco Solver utiliza-se o Microsoft Excel Solver (Figura 2Sb, material suplementar) para otimizar os valores arbitrados (concentração do ião hidrogénio em cada ponto da curva de titulação) de modo que o somatório dado pela Equação 23 tenha o valor mínimo.

No bloco Cálculos II fazem-se os cálculos do potencial da célula em cada ponto da curva de titulação usando os valores da concentração do ião hidrogénio otimizados pelo Microsoft Excel Solver e os parâmetros da calibração do eléctrodo: 


$$
E_{i}^{*}=K-S \times \mathrm{p}[\mathrm{H}]_{i}
$$

Para comparar a curva de titulação simulada com a curva de titulação experimental calcula-se o somatório dos desvios ao quadrado entre o potencial calculado e o potencial medido em cada ponto $i$ da curva de titulação:

$$
\mathrm{SDQ}=\sum_{i=1}^{\mathrm{n}}\left(\mathrm{E}_{i}^{*}-\mathrm{E}_{i}\right)^{2}
$$

Quanto menor for o valor dado pela Equação 25 melhor é o ajuste da curva de titulação simulada com a experimental e maior a confiança nos valores das constantes determinadas.

\section{PARTE EXPERIMENTAL}

\section{Reagentes e soluções}

Foi preparada uma solução de ácido nicotínico $0,100 \mathrm{~mol} \mathrm{~L}^{-1}$. $\mathrm{O}$ ácido nicotínico utilizado é da marca Acros, (grau de pureza de 99,5\%). A glicina utilizada é da marca Aldrich, e tem grau de pureza de $98,5 \%$. O ácido clorídrico utilizado foi preparado por diluição de uma ampola de 0,1 mol de ácido clorídrico, da Panreac, de forma a obter uma solução $0,100 \mathrm{~mol} \mathrm{~L}^{-1}$. A solução de hidróxido de sódio foi preparada por diluição de uma ampola de 1,0 mol de hidróxido de sódio, da Panreac, de forma a obter uma solução aproximadamente $1 \mathrm{~mol} \mathrm{~L}^{-1}$. A solução de hidróxido de sódio foi preparada em atmosfera de nitrogênio para evitar a carbonatação. A solução aquosa de cloreto de potássio, 1,0 mol L-1 , foi preparada por dissolução de cloreto de potássio, Panreac, com $99,5 \%$ de pureza. Todas as soluções foram preparadas com água desionizada ultrapura Milli-Q Plus.

\section{Instrumentação}

A solução de reagente (ácido nicotínico ou glicina) é colocada dentro de um vaso reacional de vidro da Metrohm, Ref. MTR61418220, mantido a temperatura constante por ligação a um banho termóstato F33-ME da Julabo. O vaso está tapado com uma tampa de cinco saídas nas quais são encaixados um eléctrodo de $\mathrm{pH}$ combinado Red Rod pHC2401 da Radiometer Analytical, um termómetro de resistência de platina (Pt100) ligado numa configuração de quatro fios a um multímetro Agilent 34970A, um sistema de fluxo de nitrogénio a borbulhar em contínuo na solução, uma saída de gases e uma ponta anti-difusão da Metrohm que dispensa o hidróxido de sódio para dentro da solução. O eléctrodo opera ligado a um medidor de pH PM240 da Radiometer Analytical. A solução de titulante é dispensada para dentro do vaso reacional através de uma bureta automática Multiburete 4S-D da Crison equipada com uma seringa de $1 \mathrm{~mL}$. A solução de hidróxido de sódio $\mathrm{NaOH}$ é mantida dentro de um frasco tapado com uma tampa com 3 válvulas da Omnifit, Ref. 00945Q-3V, que controlam a entrada e saída de nitrogénio e a saída para o vaso reacional. Quer o medidor de $\mathrm{pH}$ quer a bureta automática estão ligados a um computador de forma que o sistema de titulação seja controlado por um programa não comercial que lê e registra o potencial medido pelo medidor de $\mathrm{pH}$ ao longo da titulação e que dá indicação à bureta automática que seja adicionado uma alíquota de hidróxido de sódio. Só é feita nova adição de hidróxido de sódio quando a diferença entre dois conjuntos de leituras sucessivas forem inferiores a $50 \mu \mathrm{V}$.

\section{Calibração}

Antes de cada titulação do ácido nicotínico ou da glicina é realizada uma calibração do eléctrodo de vidro, para que através do potencial medido se possa conhecer a concentração do ião hidrogénio. Para a calibração adicionaram-se $1,40 \mathrm{~mL}$ de ácido clorídrico (ácido forte), $0,100 \mathrm{~mol} \mathrm{~L}^{-1}, 2,80 \mathrm{~mL}$ de cloreto de potássio, 1,0 $\mathrm{mol} \mathrm{L}^{-1} \mathrm{e}$ $25 \mathrm{~mL}$ de água desionizada ao vaso reacional. A titulação é realizada com a solução de hidróxido de sódio (base forte), $1 \mathrm{~mol} \mathrm{~L}^{-1}$, à temperatura de $20^{\circ} \mathrm{C}$ e força iónica constante de $0,10 \mathrm{~mol} \mathrm{~L}^{-1}$. Obtêm-se pares de valores, volume de base adicionado - potencial da célula (f.e.m.). Estes pares de valores são usados para obter os parâmetros da calibração do eléctrodo, a concentração rigorosa da base e o seu grau de carbonatação. Para o tratamento de resultados da calibração foi utilizado o programa GLEE. ${ }^{6}$

\section{Titulação potenciométrica}

A titulação do ácido nicotínico é realizada a $20^{\circ} \mathrm{C}$ com uma força iónica constante ao longo da titulação de $0,098 \mathrm{~mol} \mathrm{~L}^{-1}$. No vaso reaccional é adicionado $0,50 \mathrm{~mL}$ de solução de ácido nicotínico, 0,10 mol L $\mathrm{m}^{-1}, 0,50 \mathrm{~mL}$ de ácido clorídrico, 0,10 $\mathrm{mol} \mathrm{L} \mathrm{L}^{-1}$, 2,80 mL de solução de cloreto de potássio, $1,0 \mathrm{~mol} \mathrm{~L}^{-1}$ e $25 \mathrm{~mL}$ de água desionizada. Para a titulação da glicina pesam-se 0,0454 $\mathrm{g}$ de glicina directamente para a célula de titulação e adicionam-se 4,0 $\mathrm{mL}$ de cloreto de potássio, 1,0 $\mathrm{mol} \mathrm{L}^{-1}$, mais $20,0 \mathrm{~mL}$ de água e 7,8 mL de ácido clorídrico, $0,100 \mathrm{~mol} \mathrm{~L}^{-1}$. A titulação da glicina é efectuada à temperatura ambiente de aproximadamente $20^{\circ} \mathrm{C}$ e a uma força iónica média de $0,10 \mathrm{~mol} \mathrm{~L}^{-1}$. Antes de cada titulação o vaso reacional é deixado com nitrogénio a borbulhar durante 10 min sob agitação constante e só depois se inicia a titulação adicionando alíquotas de $0,005 \mathrm{~mL}$ de hidróxido de sódio, 1,01 mol L-1, para a titulação do ácido nicotínico e de $0,10 \mathrm{~mL}$ ou de $0,05 \mathrm{~mL}$ de hidróxido de sódio, para a titulação da glicina.

\section{RESULTADOS E DISCUSSÃO}

Com os dados da titulação potenciométrica do ácido nicotínico, fizeram-se os cálculos para determinar as constantes de dissociação daquele ácido, numa folha de cálculo do Excel, utilizando o algoritmo descrito, Figura 1S material suplementar. Os resultados desta titulação encontram-se resumidos na Figura 2.

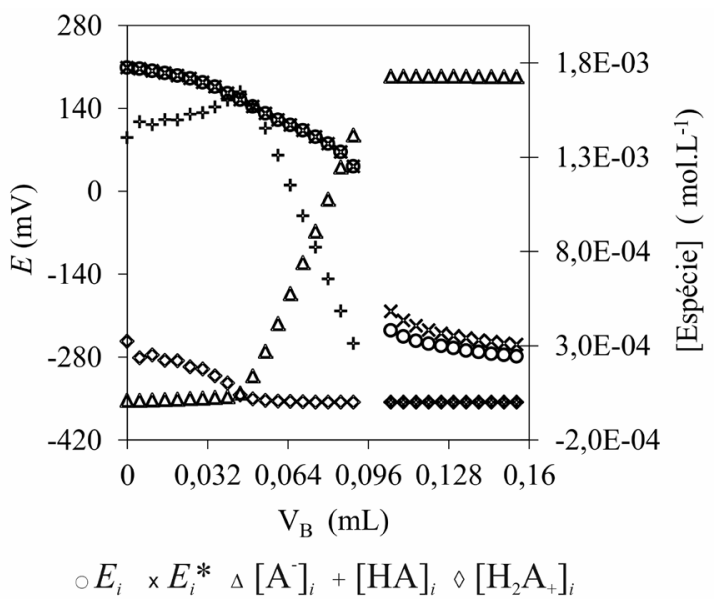

Figura 2. Valores dos potenciais observados, $E_{i}$, e calculados, $E_{i}^{*}$, na titulação potenciométrica do ácido nicotínico com hidróxido de sódio. O segundo eixo apresenta a concentração das espécies em solução

Utilizando a mesma folha de cálculo, mas com os dados da titulação potenciométrica da glicina fizeram-se também os cálculos para as constantes de dissociação deste aminoácido, que se apresentam na Figura 3. 
Tabela 1. Constantes de acidez do ácido nicotínico e da glicina obtidas, a $20^{\circ} \mathrm{C}$ e força iónica $(I)$ constante, através do tratamento dos dados das titulações potenciométricas usando o algoritmo deste trabalho, o programa Hyperquad $2008^{3}$ e os valores publicados na base de dados Mini-SCDatabase ${ }^{7}$

\begin{tabular}{lcccc}
\hline & \multicolumn{2}{c}{ Glicina $\left(I=0,10 \mathrm{~mol} \mathrm{~L}^{-1}\right)$} & \multicolumn{3}{c}{ Ácido nicotínico $\left(I=0,098 \mathrm{~mol} \mathrm{~L}^{-1}\right)$} \\
\cline { 2 - 5 } & $\mathrm{p} K_{\mathrm{a} 1}$ & $\mathrm{p} K_{\mathrm{a} 2}$ & $\mathrm{p} K_{\mathrm{a} 1}$ & $\mathrm{p} K_{\mathrm{a} 2}$ \\
\hline Algoritmo deste trabalho & $2,34 \pm 0,33$ & $9,58 \pm 0,80$ & $2,12 \pm 0,15$ & $5,00 \pm 0,16$ \\
Hyperquad2008 $^{3}$ & $2,64 \pm 0,023$ & $10,197 \pm 0,014$ & $2,286 \pm 0,078$ & $4,842 \pm 0,031$ \\
Mini-SCDatabase $^{7}$ & 2,36 & 9,76 & 1,99 & 4,71 \\
\hline
\end{tabular}

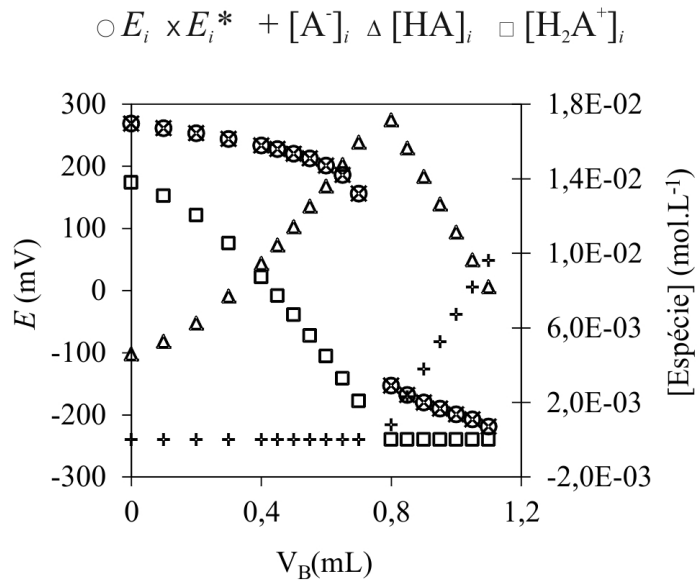

Figura 3. Valores dos potenciais observados, $E_{i}$ e calculados, $E_{i}^{*}$, na titulação potenciométrica da glicina com hidróxido de sódio. O segundo eixo apresenta a concentração das espécies em solução

Os cálculos correspondentes a estas duas titulações foram repetidos usando o Hyperquad $2008^{3}$ que é um programa comercial de utilização muito generalizada para este tipo de cálculos. Na Tabela 1 apresentamos os resultados destes cálculos e também valores para as constantes de dissociação retirados de uma base de dados. ${ }^{7}$

Tendo em conta os valores da Tabela 1 verificamos que as constantes calculadas pelo algoritmo proposto têm um erro maior que as constantes calculadas utilizando o Hyperquad $2008,{ }^{3}$ no entanto, verificamos também que as constantes calculadas pelo algoritmo proposto são coincidentes, dentro do intervalo de erro, com as constantes calculadas utilizando o Hyperquad $2008,{ }^{3}$ quer para a glicina quer para o ácido nicotínico. As constantes calculadas são concordantes com os valores apresentados na base de dados, ${ }^{7}$ exceto no caso da segunda constante de dissociação do ácido nicotínico em que se verifica um pequeno desvio, pouco significativo, quer no valor calculado usando este algoritmo como para o valor calculado através do Hyperquad2008. ${ }^{3}$

\section{CONCLUSÕES}

A experiência descrita neste artigo é rápida, envolve um procedimento experimental simples e produz bons resultados, podendo o método descrito ser aplicado a outros ácidos dipróticos.

Este algoritmo pode ser facilmente adaptado à solução dos sistemas que envolvem ácidos monopróticos, servindo neste caso como alternativa à solução através da resolução de uma equação do terceiro grau. $^{5}$

Como conclusão final podemos dizer que a folha de cálculo aqui apresentada é uma ferramenta de trabalho útil nas atividades laboratoriais que envolvam titulações potenciométricas de ácidos dipróticos. Com a folha de cálculo os estudantes podem realizar os cálculos necessários para determinar as constantes de equilíbrio e, uma vez conhecidas as constantes, podem simular as curvas de titulação usando a folha de cálculo deste programa ou programas especificamente construidos para esse fim como, por exemplo, o

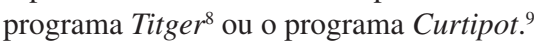

\section{MATERIAL SUPLEMENTAR}

O material suplementar está disponível em http://quimicanova. sbq.org.br, na forma de arquivo PDF, com acesso livre e é constituído pelas Figuras $1 \mathrm{~S}$ e $2 \mathrm{~S}$.

\section{AGRADECIMENTOS}

Ao Prof. M. E. Minas da Piedade (FC-UL) pela troca de ideias sobre a realização experimental e a escrita deste artigo. Este trabalho foi desenvolvido no Laboratório do Prof. M. E. Minas da Piedade (FC-UL)

\section{REFERÊNCIAS}

1. http://en.wikipedia.org/wiki/Acid_dissociation_constant, acessada em Junho 2010 e Março 2011.

2. Martell, A. E.: Motekaitis, R. J.; Determination and use of Stability Constants, $2^{\text {nd }}$ ed, VCH: New York, 1992.

3. Vacca, A.; Sabatini, A.; Gans, P.; Hyperquad2008 V5.2.9; Equilibrium Constants from Potentiometric Data; Protonic Software, Florence, 2008.

4. Gans, P.; Sabatini, A.; Vacca, A.; Talanta 1996, 43, 1739.

5. Conceição, A. C. L.; Piedade, M. E. M.; J. Chem. Educ. 2006, 83, 1853.

6. Gans, P.; O'Sullivan, B.; GLEE V3.0.21; Glass Electrode Evalution; Protonic Software, Leeds, England, 2007.

7. Powell, K. J.; Pettit, L. D.; Mini-SCDatabase; Stability Constants Database; Academic Software, 2001.

8. Oliveira, A. F.; Silva, A. F. S.; Tenan, M. A.; Olivo, S. L.; Quim. Nova 2007, 30, 224.

9. http://www2.iq.usp.br/docente/gutz, acessada em Outubro 2010 e Março 2011. 
CONSTANTES DE ACIDEZ DE ÁCIDOS DIPRÓTICOS A PARTIR DE TITULAÇÕES POTENCIOMÉTRICAS: ILUSTRAÇÃO DOS PRINCÍPIOS DO CÁLCULO ATRAVÉS DA CONSTRUÇÃO DE UM ALGORITMO MUITO SIMPLES

Elsa M. Gonçalves

Departamento de Química e Bioquímica, Faculdade de Ciências, Universidade de Lisboa, 1649-016 Lisboa / Instituto Politécnico de Setúbal, ESTBarreiro, Rua Américo da Silva Marinho, 2839-001 Lavradio, Portugal

António C. L. Conceição*

Centro de Química Estrutural, Complexo Interdisciplinar, Instituto Superior Técnico, 1049-001 Lisboa, Portugal

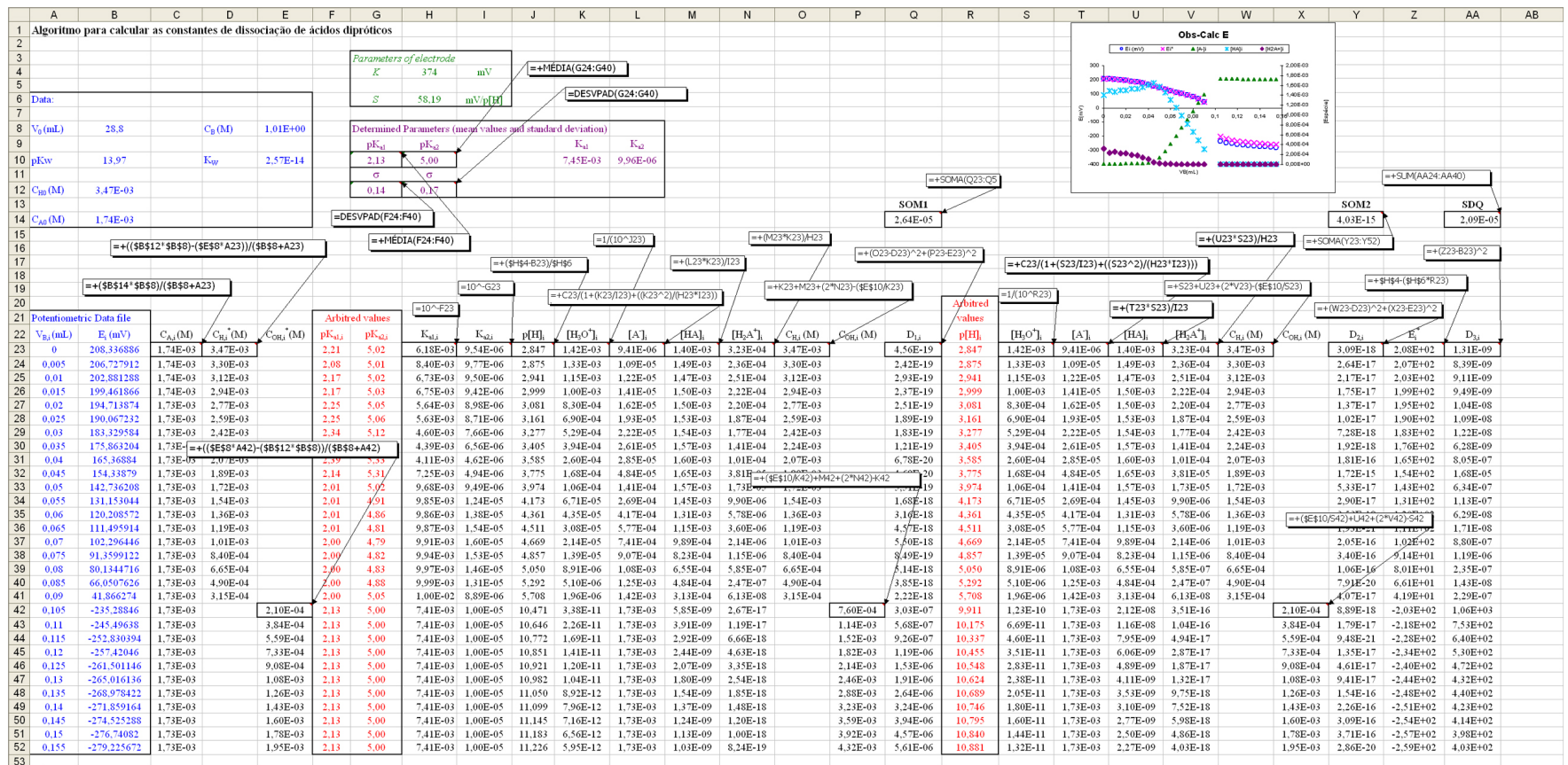

Figura 1S. Folha de cálculo do Microsoft Excel para calcular as constantes de dissociação de ácidos dipróticos a partir de curvas de titulação potenciométricas e para simular as curvas de titulação 


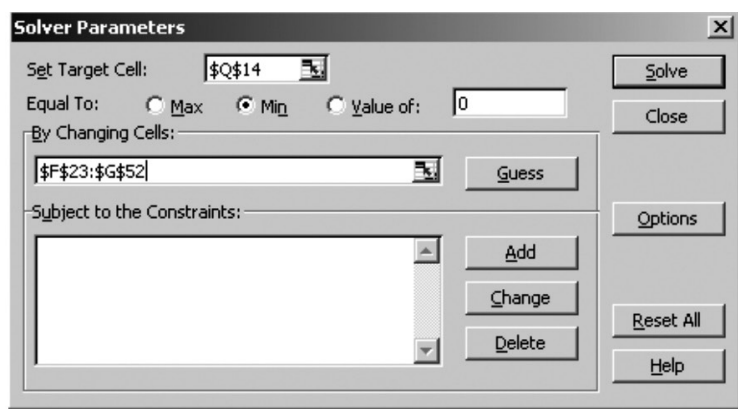

(a)

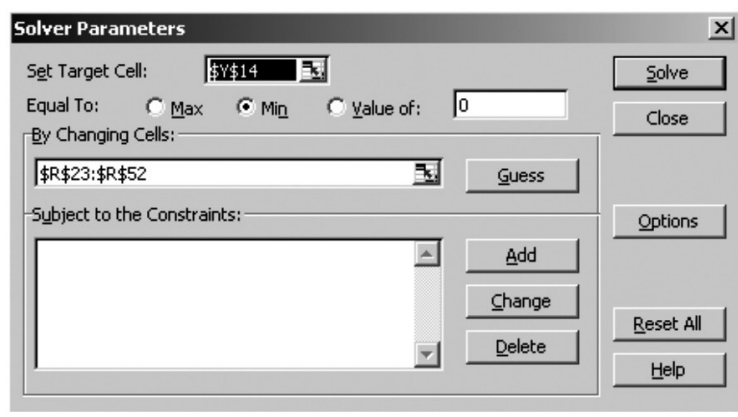

(b)

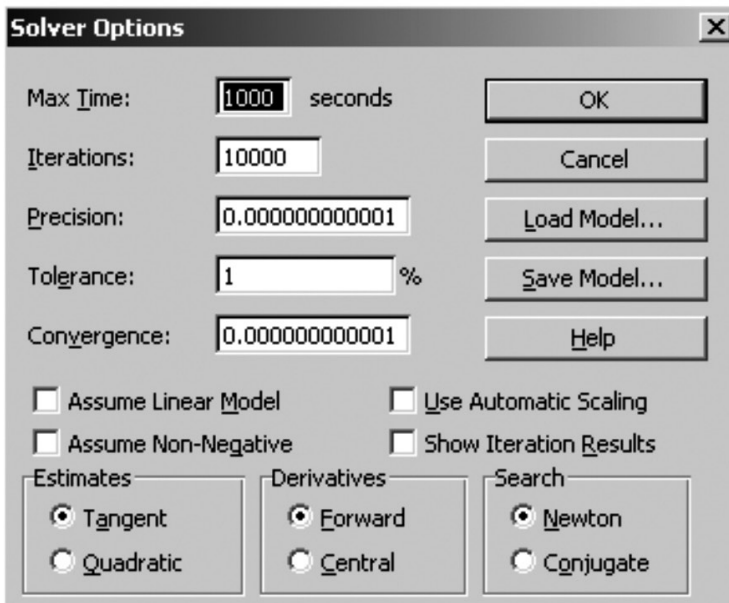

Figura 2S. Opções e parâmetros do Microsoft Excel Solver para (a) otimizar as constantes de dissociação; (b) otimizar o p[H] em cada ponto i da curva de titulação simulada 